VoL. XIX.] BEHAVIOR OF FREE ELECTRONS TOWARD GAS MOLECULES. I 73
No. 2.

\title{
BEHAVIOR OF FREE ELECTRONS TOWARD GAS MOLECULES.
}

By H. B. Wahlin.

SYNOPSIS.

Formation of Negative Ions in Gases.-The theories advanced to account for the appearance of abnormal negative mobilities at low pressures are two: it is assumed that in order to form an ion an electron must either have more than a certain amount of energy (Wellisch) or must have made, on the average, $n$ impacts (J. J. Thomson). To test the Wellisch theory, the effects of increasing the energy of the electrons was determined. The fact that the number of ions was not increased is taken to favor the Thomson theory. Assuming this theory correct the Thomson constant $n$ was determined for $\mathrm{CO}, \mathrm{NH}_{3}, \mathrm{C}_{2} \mathrm{H}_{4}, \mathrm{C}_{2} \mathrm{H}_{2}, \mathrm{C}_{2} \mathrm{H}_{6}, \mathrm{C}_{2} \mathrm{H}_{5} \mathrm{Cl}$ and $\mathrm{Cl}_{2}$, using carefully purified gases under varying conditions of pressure and frequency of alternating potential. The results depend on the values assumed for the mobility of the electron in the various gases, but they indicate that for gases in the order given, $n$ decreases progressively from about $10^{8}$ for $\mathrm{CO}$ to $\mathrm{IO}^{3}$ or. less for $\mathrm{Cl}_{2}$.

Conclusions.-CO behaves like $\mathrm{N}_{2}$; electronegative gases attach electrons more readily than do electropositive gases; the degree of saturation of hydrocarbons does not make much difference.

Mobility of negative ions in chlorine, ethane and ethylene was found by the Rutherford A.C. method to be, respectively, $0.73, \mathrm{r} .30$ and $0.9 \mathrm{I} \mathrm{cm} . / \mathrm{sec} . / \mathrm{volt} / \mathrm{cm}$. at atmospheric pressure.

\section{INTRODUCTION.}

IN order to account for the fact that the mobility of the negative ion 1 in a gas ceases to be inversely proportional to the pressure as the pressure decreases, Sir J. J. Thomson ${ }^{1}$ developed a theory in which he assumes that the electron has to make, on the average, a number of impacts, $n$, with the molecules of a gas before uniting to form an ion. If this is the case, it is clear that an electron under the action of an applied electric field will travel a greater and greater distance in the direction of the field as the pressure decreases, before combining with the molecules of the gas. Then, since the velocity of drift of the electron in unit field is great compared with that of the ion, we would expect that the mobility as measured by any of the standard methods would be abnormally high. On his assumption, Thomson has shown that out of $q_{0}$ electrons starting from a given plane in a gas the number $q$ that travel a distance $x$ without uniting with the molecules of the gas is given by

$$
q=q_{0} e \frac{-w x}{n k^{\prime} \lambda \frac{v}{d}},
$$

\footnotetext{
${ }^{1}$ Thomson, J. J., Phil. Mag ·Sept., I9I5.
} 
where $w$ is the mean velocity of agitation of the electrons, $k^{\prime}$ their mobility, $\lambda$ the mean free path and $v / d$ the applied potential gradient. In this form the equation is applicable to the Langevin method of measuring mobilities.

Loeb ${ }^{1}$ modified this expression so as to make it applicable to the Rutherford method of determining mobilities, when a square wave alternating potential is used, and obtained as an expression for the current, $I$, to the electrometer

$$
I=I_{0} e \frac{-w}{n k^{\prime} \lambda}\left[\frac{d^{2}(P / 760)^{2}}{V}-\frac{K P / 760}{N}\right],
$$

where $I_{0}$ is the value of the saturation current corresponding to a voltage $V, K^{\prime}$ and $\lambda$ are the mobility and the mean free path of the electron at a pressure of $760 \mathrm{~mm} ., K$ the mobility of the ion at this pressure, $p$ the pressure of the gas, $d$ the distance between the plates and I/ $N$ the time for a half cycle of the alternating potential. On the basis of this equation, Loeb determined the values of $n$ for $\mathrm{N}_{2}, \mathrm{H}_{2}, \mathrm{O}_{2}$, and air, assuming the mobility of the electron to be $200 \mathrm{~cm} . / \mathrm{sec} . / \mathrm{volt} / \mathrm{cm}$.

A further substitution may be made if the Townsend expression for the mobility of the electron,

$$
300 K^{\prime}=\frac{.815 e \lambda}{m w},
$$

is accepted as valid. Equation (2) then becomes

$$
I=I_{0} e \frac{-.8 \mathrm{I} 5 e}{300 n m\left(K^{\prime}\right)^{2}}\left[\frac{d^{2}(P / 760)^{2}}{V}-\frac{K P / 760}{N}\right] .
$$

This expression leaves $K^{\prime}$ as the only quantity about which there is any great degree of uncertainty. The value of $K^{\prime}$ for nitrogen has been determined by Loeb recently and has been found to be $2,800 \mathrm{~cm}$./ $\mathrm{sec} . /-$ volt $/ \mathrm{cm}$., at a pressure of $75 \mathrm{~mm}$. of mercury and in a field of 20 volts. Just what the values of $K^{\prime}$ will be in other gases, we cannot at present say, and until these values have been determined, we must make an assumption which probably only approximates the truth. This assumption is that the mobility of the free electron in a gas varies from gas to gas in the same manner as does the mobility of the negative ion. Equation (3) then becomes, when we substitute the known values for $e$ and $m$,

$$
I=I_{0} e-\frac{\mathrm{I} .9 \times 10^{8}}{n}\left(K_{n / K}\right)^{2}\left[\frac{d^{2}(P / 760)^{2}}{V}-\frac{K P / 760}{N}\right],
$$

where $K_{n}$ is the mobility of the negative ion in nitrogen.

1 Loeb, L. B., Phys. Rey., Feb.. I92 I. 
VoL. XIX.] BEHAVIOR OF FREE ELECTRONS TOWARD GAS MOLECULES. I 75
No. 2.

From this equation $n$ may be calculated from an experimental currentvoltage curve. This is done by substituting the quantities for a point on the curve in equation (4). Owing to the assumptions made in deducing this equation, it is necessary to choose a point near the asymptotic foot of the curve. The value of $n$ thus calculated is very nearly a constant, for varying frequencies of the alternating potential and varying pressures, and in the gases studied by Loeb appears to be characteristic of the gas used. The writer will, in the following pages, present evidence which will assist in showing in how far this is the case and to what extent some of the factors which might influence the attachment of electrons effect the value of $n$.

\section{Factors Influencing the Attachment of Electrons.}

There are three factors which might influence the attachment of electrons in gases:

First, an abnormal condition of the gas such as the presence of an activated form or a particular state of the molecules as regards the electronic arrangement in the outer structure. It is well known that when $\alpha$-particles or ultra-violet light passes through some gases, changes take place. For example, in oxygen we have ozone $\left(\mathrm{O}_{3}\right)$ formed and it is possible that this might attach the electrons more readily than do the $\mathrm{O}_{2}$ molecules. Now, in general, the amount of activation when ultraviolet light is used to produce the electrons would be comparatively small, but if an electron attaches at every impact with these activated molecules, the effect of a small amount of the impurity would be to change the result greatly. However, in some of the determinations made, the writer varied the intensity of the ultraviolet light by a factor of four with no noticeable change in the readiness with which the electrons attach. Where $\alpha$-particles are used as a source of ionization, it is possible that enough complete chemical change would be produced to influence the value of $n$ for a gas very markedly.

The second possibility is that the electrons must strike the molecules in a certain place in order to attach.

The third factor that might influence the attachments is the relative energy of the electron and molecule at impact. For in the light of our present knowledge of atomic structure, it is conceivable that the electron must have a certain critical energy of approach in order to combine with the molecule. The question which we must decide, then, is whether or not the mean energy of thermal agitation is sufficient to cause attachments and whether or not an increase of the energy of the electrons above that of agitation will influence the constant of attachment, $n$. This 
question can be answered experimentally as the following considerations will show.

The Effect of Energy on the Attachment of Electrons.

Wellisch, ${ }^{3}$ using the Franck modifications of the Rutherford method for measuring mobilities, found below a pressure of $8 \mathrm{~cm}$. in air, evidence for two types of carriers of negative electricity. One type showed the normal variation of mobility with pressure. The other gave abnormally high mobilities with decreasing pressures. Carriers of this second type, Wellisch considered to be free electrons, and he accordingly concluded that the negative carriers in a gas consist of normal ions and permanently free electrons. To account for these free electrons, he assumed that the energy of the electron at impact must exceed a certain value in order to combine to form an ion. To account for the two kinds of carriers observed, the energy would have to exceed the mean energy of thermal agitation, and Wellisch assumed that this additional energy required comes from the energy of emission of the electrons. In short, the explanation he offered was that in order to make an attachment, the electron must strike another molecule within a certain distance of the parent molecule; i.e., before its kinetic energy of emission has decreased below the critical value necessary to form an ion.

If this explanation is to hold, we should expect that in the Wellisch experiment, there would be no change in the nature of the mobility curves obtained with increasing values of the field driving the electrons through the gauze, until the velocity acquired in the field became comparable with the velocity of emission of the electrons from the molecules under the influence of $\alpha$-rays. For a small change in the field would not materially alter the chance an electron has of striking a molecule within a certain distance of the molecule from which it comes, the velocity of emission being great compared with the velocity acquired in the field. That the curves actually do change their form for relatively small changes in the field was shown by Loeb, ${ }^{2}$ who found that as the auxiliary field is increased, the evidence for the two types of carriers disappeared entirely. This indicates weakness in the Wellisch theory.

A further test of this theory may be made by increasing the energy of the electrons in the auxiliary field to a higher value than was used in the experiments by Loeb, for it is to be expected on the basis of the Wellisch theory that before the electron gains ionizing energy in the field, the number of free electrons passing through the gauze would fall to zero, because the energy acquired by the electrons between impacts would

3 Wellisch, E. M., Am. Journal of Science, 4, 39, p. 583, I9I 5 . Am. Journal of Science, 4, 44 , p. I, I9I 7 . 
VoL. XIX.] BEHAVIOR OF FREE ELECTRONS TOWARD GAS MOLECULES. I 77
No. 2.

then exceed the energy of emission from the molecules when the gas is ionized by $\alpha$-particles. The electrons would therefore be making impacts with the molecules equal to or exceeding the energy necessary for attachment.

In order to determine whether this is the case, the writer converted the apparatus as used by Loeb in his determination of $n$, to one of the Wellisch type, the only difference being that whereas Wellisch used a radioactive substance to produce his ionization, the writer obtained his photoelectrically, by letting ultra-violet light strike a speculum metal plate. Fig. I. $(a)$ is a plate of speculum metal; $(b)$ is a battery which

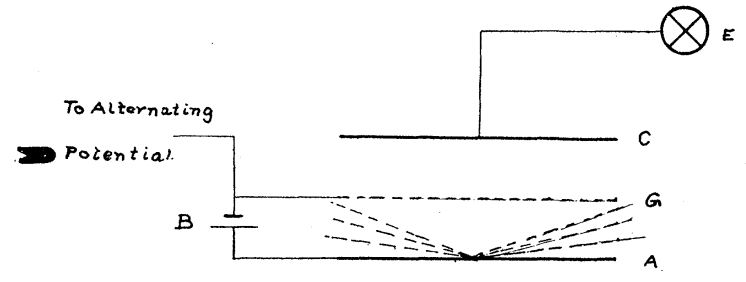

Fig. 1.

produces the field for driving the electrons and ions through the gauze $(g)$. $(c)$ is a collecting plate $\mathrm{I} 0 \mathrm{~cm}$. in diameter connected to the electrometer $(e)$. The alternating potential is connected to the gauze. 'The distance from $(a)$ to $(g)$ was made $\mathrm{I} .5 \mathrm{~cm}$. and the distance from $(g)$ to $(c) \mathrm{I} .3 \mathrm{~cm}$. The gauze was made by boring holes I $\mathrm{mm}$. in diameter in a sheet of brass one half $\mathrm{mm}$. in thickness, the distance between adjacent holes being about I $\mathrm{mm}$. With this gauze the writer encountered no difficulty due to the stray field carrying a charge to the collecting plate. Tests were made for a stray field by varying the distance between the collecting plate and the gauze. The current corresponding to a given value of the alternating potential was measured by noting the charge acquired by the collecting plate in a given time. In all this work air was used at pressures of $8 \mathrm{~cm}$. and below. With pressures of from 4 to $8 \mathrm{~cm}$., and with values of the auxiliary field of from 3 to 15 volts $/ \mathrm{cm}$., the two types of carriers noted by Wellisch were observed.

As is shown in Curve I. (Fig. $2 A$ ), with an auxiliary field of 9 volts and at a pressure of $4 \mathrm{~cm}$., the two types of carriers observed by Wellisch are present. With an auxiliary field of 150 volts (II., Fig. $2 A$ ), the evidence for the two kinds disappears entirely. If the carriers were the normal ions only, this curve as well as III. and IV. in Fig. $2 B$, which were obtained with auxiliary field of 640 and I,400 volts respectively, should strike the voltage axis at about 35 volts. The curves do change 
their form in that the current corresponding to a given voltage decreases for high values of the auxiliary fields. This, however, cannot be taken as evidence for an increase in the number of ionic carriers, as the saturation currents taken with a steady potential showed this same tendency.

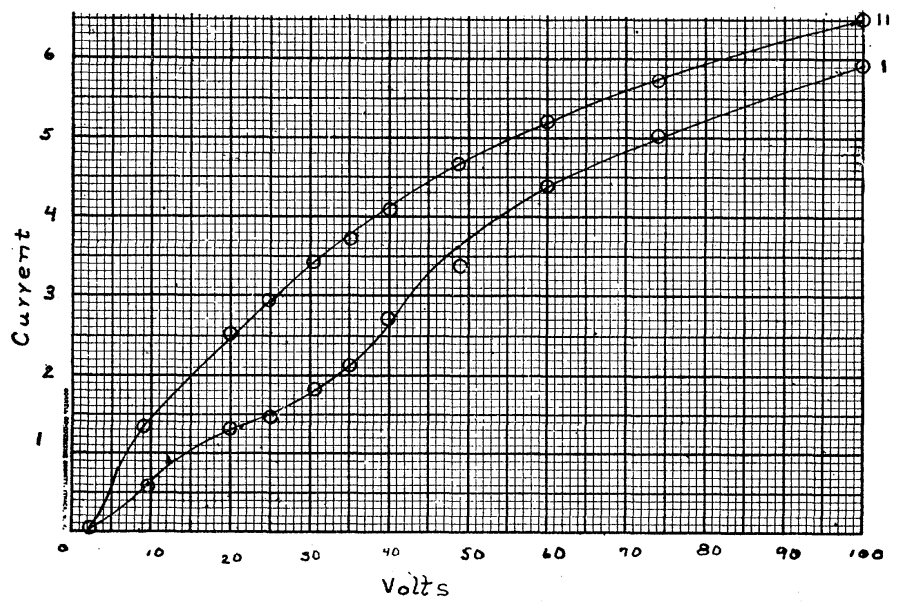

Fig. $2 A$.

Pressure $4 \mathrm{~cm}$. Frequency of alternating potential, 7 ro alternations per sec. I.- -auxiliary field -9 volts. II.- - auxiliary field $-\mathrm{I} 50$ volts.

We see from these results that an increase in the energy of the electrons above that of thermal agitation does not appreciably influence the number of attachments, and must conclude that the mean energy of agitation at ordinary temperatures is sufficient to cause the electrons to combine with the molecules. This makes the Wellisch theory untenable and leaves as the only alternative which we at present have, the Thomson theory.

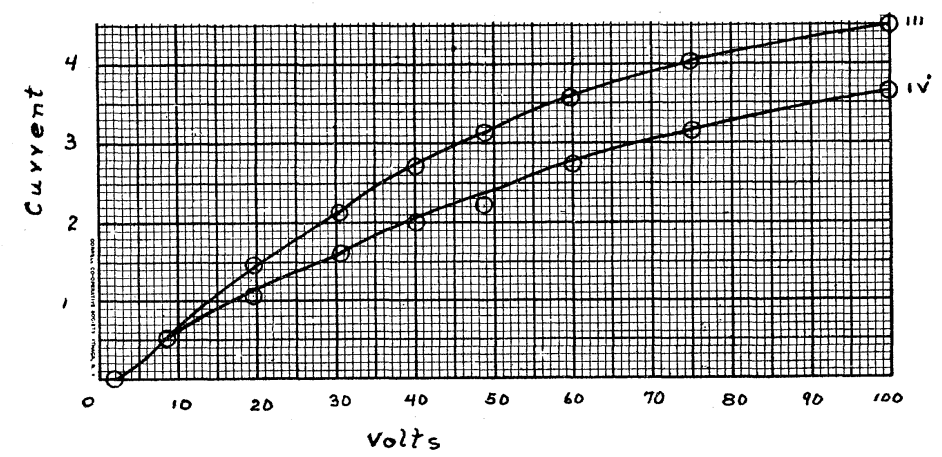

Fig. $2 B$.

Pressure $4 \mathrm{~cm}$. Frequency of alternating potential 7 Io alternations per sec. III.auxiliary field of 640 volts. IV.--auxiliary field of $\mathrm{r}, 400$ volts. 
Vol. XIX.] BEHAVIOR OF FREE ELECTRONS TOWARD GAS MOLECULES. I 79
No. 2.

It next becomes of interest to determine the constant, $n$, of the Thomson theory to see how it varies in different gases with the chemical nature of the gases and with the different types of chemical bonds between the atoms of the molecules of which the gas is composed. Results of this kind may furnish data that will help us to a better understanding of outer atomic and molecular structure.

\section{Determination of the Thomson Constant.}

In general, the method used in the determination and calculation of $n$ was the same as that used by Loeb in his experiments. The writer has found the value of $n$ for seven gases; viz., $\mathrm{CO}, \mathrm{NH}_{3}, \mathrm{C}_{2} \mathrm{H}_{2}, \mathrm{C}_{2} \mathrm{H}_{4}, \mathrm{C}_{2} \mathrm{H}_{6}$, $\mathrm{Cl}_{2}$ and $\mathrm{C}_{2} \mathrm{H}_{5} \mathrm{Cl}$. The determinations were made on at least five different samples of each of the gases, and a number of mobility curves under varying conditions of pressure and frequency of the alternating potential were obtained from each sample. In most cases the pressure variation ranged from $7.6 \mathrm{~cm}$. to $76 \mathrm{~cm}$. and the frequency from I 45 alternations $/ \mathrm{sec}$. to 712 alternations/sec. Whenever possible, the mobility of the negative sion was determined, and if this could not be done, the values found by other observers were used. In making a series of determinations, the chamber was washed out four or five times with the gas being used, by filling to atmospheric pressure, exhausting to a pressure of $\mathrm{I} .5 \mathrm{~cm}$., and filling again. Thus the residual gas from any previous determinations was very small. The value of $n$ was calculated from ten to twelve of the most representative curves for each gas.

In the preparation of the gases, considerable care was taken to get them pure. The importance of this is shown in the work on hydrogen by Loeb. He obtained electronic mobilities in this gas at atmospheric pressure, where other observers had been able to get comparatively low mobilities only. Furthermore, the method of preparation chosen for each gas was such that any impurity present would cause only an unappreciable number of attachments, for obviously the presence of a small percentage of an impurity which attaches electrons readily would change the value of $n$ far more than one which does not. The method of preparation and purification of each gas follows:

\section{Preparation and Purification of Gases.}

Carbon monoxide was prepared by the action of concentrated sulphuric acid on sodium formate. The gas evolved was bubbled through a solution of sodium hydroxide in a tower packed loosely with glass wool, which served to break up the bubbles and retard their progress through the solution. The gas was then passed over calcium chloride to remove 
any excess of water vapor and over hot copper to break up any organic impurity and remove free oxygen. If there were a trace of oxygen present, there would be some carbon dioxide formed at this point, and in order to remove this, the gas was passed over solid sodium hydroxide. It was then passed over calcium. chloride and phosphorous pentoxide to complete the drying, through two traps cooled to liquid air temperatures and finally into the chamber. The cooling served to remove the last trace of any of the more easily condensible impurities. Density tests on the gas checked with the true value to within one part in two thousand.

Ammonia was prepared by heating a solution of ammonium. chloride and sodium hydroxide to boiling. A reflux condenser connected to the generating flask removed the excess of water vapor. From this the gas passed over calcium oxide to remove any water remaining and was next liquified in a trap cooled to $-\mathrm{I}$ I $2^{\circ}$ with frozen alcohol. The liquid ammonia was boiled under reduced pressure to remove oxygen and any of the more volatile impurities, and the liquid thus purified was allowed to vaporize in to the chamber first passing through a trap cooled to $-26^{\circ}$ to remove vapors of stopcock greases.

Acetylene was prepared by the action of water on calcium carbide. The gas was first passed through a series of wash bottles containing solutions of cupric sulphate made slightly acid with sulphuric acid. This removed hydrogen sulphide and phosphine, which are the chief impurities present in acetylene prepared from the commercial carbide. The gas then passed over calcium. chloride for a preliminary drying, through a trap cooled to $-26^{\circ}$ with frozen carbon tetrachloride and was condensed in a second trap cooled with frozen alcohol. About one third of the condensed acetylene was evaporated under reduced pressure. The next third was passed over calcium chloride and phosphorous pentoxide, through a trap cooled to $-26^{\circ}$, and into the chamber. The remaining third of the gas escaped to the air directly. By this method of fractionation, the less volatile as well as the more volatile impurities would be removed.

Ethylene was prepared by the common laboratory method; i.e., by heating a mixture of concentrated sulphuric acid and ethyl alcohol to a temperature of $165^{\circ}$. The gas evolved was passed through water to remove any alcohol that would be carried over, and through solutions of sodium hydroxide to remove any sulphur dioxide and sulphur trioxide fumes. The water and sodium hydroxide solutions would also remove the greater part of the ether that is evolved in the process. Next the ethylene passed over calcium chloride, then through a trap cooled to $-85^{\circ}$ with frozen methyl alcohol, and was condensed in a second trap 
VoL. XIX.] BEHAVIOR OF FREE ELECTRONS TOWARD GAS MOLECULES. I 8 I
No. 2.

cooled to - $112^{\circ}$ with frozen ethyl alcohol. The gas was fractionated and dried by the same method used for acetylene, and then passed through a trap cooled to $-85^{\circ}$ into the chamber.

Ethane ${ }^{1}$ was prepared by the electrolysis of a concentrated solution of sodium acetate in water, the solution being made slightly acid with acetic acid. In the process, hydrogen is given off at the cathode and a mixture of ethane and carbon dioxide at the anode. Care was taken to keep the solution cool during the electrolysis as the efficiency of the process decreases very much with an increase in temperature. Some acetic aldehyde is formed due to the reducing action of the hydrogen, but this, together with the carbon dioxide liberated, was removed by passing the gas through a number of solutions of sodium hydroxide. The process of drying and fractionating was the same as that used for acetylene. Before entering the chamber the gas passed through a trap cooled to $-28^{\circ}$.

Ethyl chloride was obtained in a pure state from the tubes used by the medical profession for freezing purposes, but was passed through a dilute.solution of sodium hydroxide to remove hydrochloric acid gas and then through a solution of sodium thiosulphate to remove any free chlorine that might be present. It was then condensed in a trap cooled to $o^{\circ}$. The process of fractionation and drying was the same as that used for acetylene.

Chlorine was obtained from a tank kindly furnished by Dr. G. L. Wendt, of the Department of Chemistry. During the first two fillings the gas was condensed and fractionated and then dried by passing it over phosphorus pentoxide before it entered the chamber. It was found advisable, however, to dispense with the fractionation, since the gas reacted so rapidly with the metallic surfaces of the chamber that it was necessary to make a filling and take the data in as short a time as possible.

\section{Determination of $n$ For Air and Chlorine.}

The writer was unable to get any appreciable photoelectric emission in the presence of chlorine. Although a number of metallic surfaces of different materials were tried, in no case was the emission great enough to be of any use in this experiment. The apparatus was, therefore, converted into one of the Wellisch type, and the ionization was produced by $\alpha$-particles from polonium. The polonium was deposited on a copper plate which was fastened to the lower side of the gauze $(g)$,

1 This method is preferable for this experiment to the accepted one for preparing pure ethane; viz., by the action of a zinc-coppered couple on an alcoholic solution of ethyl iodide, since the presence of a trace of any of the halogens as an impurity would alter the results enormously. 
(Fig. I). Care was taken so as to shield the upper part of the chamber from the radiation.

In order that this method shall be applicable to the Thomson theory, the calculations must be made from curves obtained at pressures so low that at least a portion of the electrons liberated pass through the gauze before combining with the molecules of the gas. In other words, the curves must be such as to show the two types of carriers noted by Wellisch. To determine to what extent the method is applicable to the Thomson theory, a series of determinations of $n$ for air was first made. Fig. 3

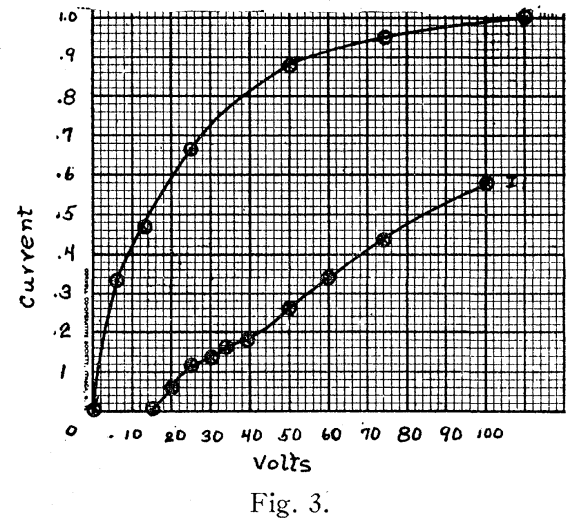

Pressure $7.5 \mathrm{~cm}$. Frequency of alternating potential $7 \mathrm{I} 2$ alternations per sec. Auxiliary field-6.o volts. I.-Mobility curve. II.-Saturation curve. The current is given in fractional parts of the saturation current at Ioo volts.

illustrates the type of curve obtained at a pressure of $7.5 \mathrm{~cm}$. The lower part of this curve; i.e., below 42 volts, is due to electrons which get through the gauze before combining. These then start from the gauze as do the photoelectrons in the other determinations of $n$, and it is therefore from this portion of the curve that our calculations must be made. In this case, the value of $I_{0}$ cannot be taken as the value of the saturation current corresponding to the voltage in question. For, obviously, the saturation current is made up of two parts-one part of the current being due to the carriers which start from the gauze as ions and the other part being due to those carriers which start from the gauze as electrons. It is this latter part which determines the value of $I_{0}$ in equation 4 . Since there is no way in which a distinction between the two types can be made in the case of the saturation current, an approximation to the true value was obtained by assuming $I_{0}$ to be equal to the value of the mobility current at the voltage in each case where the electronic carriers predominate. In Fig. 3 this would correspond to the value at 42 volts. However, since the calculations must be made from the lower parts of 


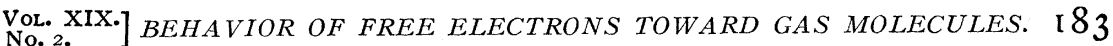

the mobility curves, it is necessary to correct this value further for the change in the saturation current as the voltage decreases. So that the value of $I_{0}$ thus obtained must be multiplied by the ratio of the saturation current at the voltage in question to the value of the saturation current at the voltage at which $I_{0}$ is chosen. In general, a determination thus made would give too low a value for the saturation current, but since in the determination of $n$, only the logarithm of $I / I_{0}$ enters, the error would not be enough to change the results by an appreciable factor. The value of $n$ thus calculated was found to be $4 . \mathrm{I} \times \mathrm{IO}^{4}$ which compares very favorably with the value of $4.3 \times 1 \mathrm{I}^{4}$ as found by Loeb.

No abnormal mobilities could be obtained in chlorine even at pressures of $\mathrm{I} .3 \mathrm{~cm}$. The writer could not work at pressures below this for the ionization produced by the $\alpha$-particles became so small that the currents were difficult to measure. However, an upper limiting value of $n$ may be calculated if it is assumed that the least ration of $I / I_{0}$ that could be measured is .03. Then for the voltage just below the value at which the mobility curve strikes the voltage axis, and for values of the pressure, frequency, and plate distance corresponding to the conditions under which the curve was obtained, a value of $n$ was determined. This will be given in the table below.

Table of Results.

\begin{tabular}{|c|c|c|c|c|c|}
\hline Gas. & from Equation & $\begin{array}{c}K \\
\text { Ionic } \\
\text { Mobility. }\end{array}$ & from Equation & $\begin{array}{c}\text { Limits of } \\
\text { Variation of } n \\
\text { in Equation (2). }\end{array}$ & Observer. \\
\hline $\mathrm{N}_{2}$. & Infinite & 2.50 & Infinite & - & Loeb \\
\hline $\mathrm{H}_{2}$. & Infinite & - & Infinite & 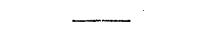 & Loeb \\
\hline $\mathrm{CO} \ldots$ & $1.6 \times 10^{8}$ & 1.20 & $3.1 \times 10^{7}$ & $(1-6) \times 10^{7}$ & Wahlin \\
\hline $\mathrm{NH}_{3} \ldots$ & $9.9 \times 10^{7}$ & 0.78 & $1.3 \times 10^{7}$ & $(.7-1.6) \times 10^{7}$ & Wahlin \\
\hline $\mathrm{C}_{2} \mathrm{H}_{4} \ldots$ & $4.7 \times 10^{7}$ & 0.91 & $7.1 \times 10^{6}$ & $(.18-1.6) \times 10^{7}$ & Wahlin \\
\hline $\mathrm{C}_{2} \mathrm{H}_{2}$. & $7.8 \times 10^{6}$ & 1.15 & $1.5 \times 10^{6}$ & $(0.8-2.3) \times 10^{6}$ & Wahlin \\
\hline $\mathrm{C}_{2} \mathrm{H}_{6}$. & $2.5 \times 10^{6}$ & 1.30 & $5.3 \times 10^{5}$ & $(2.5-8.8) \times 10^{5}$ & Wahlin \\
\hline $\mathrm{CO}_{2}{ }^{1}$. & $1.5 \times 10^{7}$ & 1.22 & $2.9 \times 10^{6}$ & $(2.3-4.3) \times 10^{6}$ & Loeb \\
\hline $\mathrm{CO}_{2}{ }^{2} \ldots$ & $3.5 \times 10^{6}$ & 1.22 & $6.9 \times 10^{5}$ & & Loeb \\
\hline $\mathrm{CO}_{2}{ }^{3}$. & $2.1 \times 10^{5}$ & 1.22 & $4.0 \times 10^{4}$ & & Loeb \\
\hline $\mathrm{N}_{2} \mathrm{O}^{4}$. & $6.1 \times 10^{5}$ & 1.33 & $1.4 \times 10^{5}$ & $(0.8-2.3) \times 10^{5}$ & Loeb \\
\hline $\mathrm{N}_{2} \mathrm{O}^{5}$. & $3.6 \times 10^{5}$ & 1.33 & $7.8 \times 10^{4}$ & $\ldots$ & Loeb \\
\hline $\mathrm{C}_{2} \mathrm{H}_{5} \mathrm{Cl}$. & $3.7 \times 10^{5}$ & 0.30 & $1.8 \times 10^{4}$ & $(1.3-2.8) \times 10^{4}$ & Wahlin \\
\hline $\operatorname{Air}^{6}$. & $4.1 \times 10^{4}$ & 2.50 & $1.7 \times 10^{4}$ & $(0.7-5.7) \times 10^{4}$ & Wahlin \\
\hline Air. & $4.3 \times 10^{4}$ & 2.50 & $1.8 \times 10^{4}$ & $(0.7-6.4) \times 10^{4}$ & Loeb \\
\hline $\mathrm{O}_{2} \ldots$ & $8.7 \times 10^{3}$ & 2.50 & $3.6 \times 10^{3}$ & $(1.5-5.7) \times 10^{3}$ & Loeb \\
\hline $\mathrm{Cl}_{2} \ldots$ & $\begin{array}{l}\text { Less than } \\
2.1 \times 10^{3}\end{array}$ & 0.73 & $\begin{array}{c}\text { Less than } \\
240\end{array}$ & - & Wahlin \\
\hline
\end{tabular}

${ }^{1}$ Freshly prepared.

24 hours old.

${ }^{3} 22$ hours old.

${ }^{4}$ Freshly prepared.

524 hours old.

${ }^{6}$ Using $\gamma$-particles. 
Results of Determination of $n$.

The table above gives the values for the mobility of the negative ion used in the calculations of $n$. It also gives the value of $n$ as calculated from equation 2 and from equation 4 , assuming electronic mobility to be proportional to the mobility of the negative ion in both cases.

The values given for the mobility of the negative ion are in most cases higher than the generally accepted values, but the Rutherford method of measuring mobilities gives results which in general are higher than those obtained by other methods. As far as the writer has been able to learn, this is the first time the mobility of the negative ion in ethane, ethylene and chlorine have been determined. Rutherford, however, measured the sum of the mobilities of the positive and negative ions in chlorine and found for this value $2 \mathrm{~cm}$. per sec. per volt per $\mathrm{cm}$. The results on ethane and ethylene were obtained using the Rutherford method and those on chlorine by using the Franck modification of this method.

\section{Discussion of Results.}

As will be seen from these results, the values for $n$ calculated on the basis of equation (4) do not agree with those from equation (2), assuming in each case the mobility of the electron to be proportional to that of the ion and independent of the pressure and field strength. Unfortunately our data on electronic mobility are as yet too meager to tell us which set of values most nearly approximates the truth, but whichever set is chosen, the values will serve for comparison of the relative electronic affinites for different gases.

In most cases the value of $n$ is high, showing that the electron does not readily attach itself to a molecule. The fact that in no case is the variation for any gas outside the limits of experimental error indicates that the value of $n$ for a gas is characteristic of that gas. This is further indicated by the fact that the value found for air, using the Franck modification of the Rutherford method and using $\alpha$-particles as the ionizing source, checks with Loeb's result which he obtained producing the electrons photoelectrically.

It is highly probable that the electron must have a certain critical energy of approach in order to combine to form an ion, but as has been shown, this critical energy is less than the mean energy of thermal agitation, since an increase of the energy of the electrons above that of agitation does not measurably influence the attachments. This leaves two alternatives in explanation of these results; viz., either the electron must strike the molecule in some particular place, or the electron must strike the molecule in some particular state. Whichever explanation holds, 
VoL. XIX.] BEHAVIOR OF FREE ELECTRONS TOWARD GAS MOLECULES. I 85
NO. 2.

the Thomson theory is still valid, so we have no means of differentiating between the two from the results above.

The fact that $n$ for air is about 5 times the value for $\mathrm{O}_{2}$ indicates that it is to the $\mathrm{O}_{2}$ molecule in air that the electron attaches. This would also be indicated by the fact that the $\mathrm{N}_{2}$ and $\mathrm{H}_{2}$ molecules do not attach electrons. It is not surprising that these gases give no attachments, for the reluctance the molecules of these gases show for acquiring a negative charge has already been observed by methods of positive ray analysis. J. J. Thomson in his "Positive Rays" states that in no case has a negatively charged nitrogen molecule ever been observed, and only rarely a negatively charged hydrogen molecule.

On account of the resemblance between nitrogen and carbon monoxide as regards a number of their chemical and physical properties, Langmuir ${ }^{1}$ and more recently, Thomson ${ }^{2}$ have assigned to the molecules of the two gases the same outer molecular structure. Thomson further points out that we should therefore expect a similarity in behavior between the two as regards electronic attachments. As may be seen from the value of $n$ for $\mathrm{CO}$, only rarely does an electron attach to form an ion. In fact, it is possible that it never does. The writer was able to get mobilities for the negative ion in $\mathrm{CO}$ of $240 \mathrm{~cm}$./sec. per volt per $\mathrm{cm}$., and although on the Thomson theory this has no significance as a mobility measurement, it will serve to show that the electron will remain free in the gas an appreciable length of time. Such high mobilities have never been obtained before. In the case of $\mathrm{NH}_{3}$ it is to be expected that the electron will not attach readily, for the $\mathrm{NH}_{3}$ molecule tends to attach a positively charged hydrogen ion whenever possible and should therefore not show a high degree of electronic affinity.

The results on acetylene, ethylene, and ethane show that as far as the attachments are concerned, there is very little difference between molecules having a double, triple, or a single bond. In a recent paper on the structure of molecules and chemical combinations, J. J. Thomson states that the attachment of an electron to a molecule having a single bond between the atoms might sufficiently loosen the contacts between the atoms so that the molecules would break up. Unfortunately a determination of the mobility of the ion in a gas furnishes us no means for determining if this is the case, since, as has been shown by Wellisch ${ }^{3}$ and Franck,${ }^{4}$ the mobility of carriers of atomic dimensions is independent of the size of the carrier. Although it is possible that in some cases the

${ }^{1}$ Langmuir, Irving, Jour. Am. Chem. Soc., 4I, 6, p. 868, I9I9.

2 Thomson, J. J., Phil. Mag., March, I92r.

${ }^{3}$ Wellisch, E. M., Proc. Roy. Soc., 82A, 509, 1909.

${ }^{4}$ Franck, 9 Ber. d. Dtsch. Phys. Ges., II, 397, I909. 
attachment of an electron will cause the molecule to split up into a negatively charged atom and a neutral one, it does not seem possible that in a comparatively inert gas like ethane, in which the bond between the two carbon atoms remains intact in most chemical reactions if not in all, the energy of the electron at impact would be sufficient to cause the molecule to break up into a negatively charged atom and a neutral $\mathrm{CH}_{3}$ group.

The aging effect observed by Loeb in carbon dioxide is as yet unexplained. He prepared the gas by treating pure marble with hydrochloric acid. The writer made a number of determinations in which the gas was prepared by heating sodium bicarbonate, but was unable to get any aging effect. The value of $n$ in this case was more nearly equal to the lowest value in the table above; i.e., to the value after aging. However, the gas in this instance was purified by fractionation and it is possible that in the process of condensation and evaporation, the effect is lost. Loeb also noticed a slight change in $\mathrm{N}_{2} \mathrm{O}$ with time and explained it as being due to a dissociation of the gas into $\mathrm{N}_{2}$ and $\mathrm{O}_{2}$.

The comparatively high degree of electronic affinity of $\mathrm{O}_{2}$ and $\mathrm{Cl}_{2}$ is not surprising, since it is to be expected that the electronegative elements; i.e., those which tend to gain electrons, should show a greater number of attachments than do the electro-positive elements. Furthermore, chlorine, being the more electro-negative of the two, shows the greater tendency to form a negative ion.

That the degree of electronic affinity is determined by the atoms of the molecule rather than by the bonds between the atoms is indicated by the results on ethyl chloride. For by a comparison of the results on this gas with the results on ethane, we see that the replacement of one of the hydrogen atoms with one of chlorine increases the number of attachments appreciably.

This problem was assigned by Dr. R. A. Millikan and was carried out under the directions of Dr. L. B. Loeb. The author wishes to express his thanks to these gentlemen for suggestions and criticisms throughout the progress of the work. 